\title{
Amelioration Du Systeme De Management De Qualite Via Une Contribution A La Mise En Place D'un Systeme De Management De Securite Dans Une Societe Multinationale Au Maroc
}

\author{
Aziz Bahlouli \\ Chaouch Abdelaziz \\ Laboratory of Biotechnology, Environment and Quality (LABEQ), \\ Department of Biology, Faculty of Science, \\ Ibn Tofaïl University, Kenitra, Morocco \\ Ghizlane Hajjaj \\ Laboratory of Pharmacology and Toxicology, Department of Drugs \\ Sciences, Faculty of Medicine and Pharmacy, Mohammed V University, \\ ERTP, Rabat Instituts, Agdal, Rabat, Morocco

\section{Mahjoub Aouane} \\ Laboratory of Biotechnology, Environment and Quality (LABEQ), \\ Department of Biology, Faculty of Science, \\ Ibn Tofaïl University, Kenitra, Morocco
}

Doi:10.19044/esj.2018.v14n31p416 URL:http://dx.doi.org/10.19044/esj.2018.v14n31p416

\begin{abstract}
Managing staff security has become a global requirement and challenge. Moroccan companies are obliged to guarantee the safety of their personnel through a security management system. The implementation of the SMS (Safety Management System) has now become a regulatory requirement. This article presents a study carried out in a multinational company, a production and packaging unit for fresh fruits and vegetables, with a view to setting up an SMS, in an approach aimed at improving the quality system of the company.
\end{abstract}

Keywords: Quality Management System, security, Environment, MacroProcess Standards.

\section{Résumé}

La maîtrise de la sécurité de personnel est devenue une exigence et un enjeu à l'échelle mondiale. Les entreprises marocaines, sont tenues de garantir la sécurité de leur personnel par le biais de système de management sécurité. 
La mise en place du SMS (Système de management de sécurité), est désormais devenue une exigence réglementaire. Cet article présente une étude effectuée dans une entreprise multinationale, unité de production et de conditionnement des fruits et des légumes frais, en vue de mettre en place un SMS, dans une démarche visant l'amélioration de système qualité de l'entreprise.

Mots clés : Système de management de la qualité, Sécurité, Environnement, Normes Macro-processus.

\section{INTRODUCTION}

Depuis longtemps, les grandes entreprises ont mis en place leur référentiel sécurité en utilisant le concept du système de management et les principes d'amélioration continue. Depuis une dizaine d'années, les motivations des entreprises pour la mise en place d'un système de management de la santé et de la sécurité au travail rejoignent celles qui sont exprimées pour la mise en place des systèmes de management de la qualité et environnement. Un système de management de la santé et de la sécurité au travail (SMS) est une partie du système de management global de l'entreprise. L'adoption d'un tel système est l'expression d'une approche globale et gestionnaire de la prévention des risques professionnels. Elle se base sur un référentiel et suit une démarche de changement qui doit être animée et soutenue. Les premiers constats effectués dans quelques entreprises montrent que les résultats sont contrastés : ils dépendent plus de l'utilisation du système de management que de son choix. La mise en œuvre d'un SMS est recommandée pour autant qu'un certain nombre de valeurs essentielles et bonnes pratiques de prévention soient adoptées.

La maîtrise de la sécurité alimentaire est devenue une exigence et un enjeu à l'échelle mondiale. Les entreprises agricoles marocaines, sont tenues de garantir la sécurité alimentaire de leurs produits par le biais de système de management qualité approprié. La mise en place du système $\mathrm{HACCP}^{1}$ (Hazard Analysis and Critical Control Points), système d'analyse des risques et maîtrise des points critiques, est désormais devenu une exigence réglementaire c'est pour cette raison que cette société qu'on a choisi pour cette étude à procéder à mettre en place la certification BRC (British Retail Consortium) et IFS (International Food Standard), ça après avoir obtenu la certification Global Gap ${ }^{2}$.

\section{ETUDE BIBLIOGRAPHIQUE SUR LE SYSTEME DE MANAGEMENT DE LA SECURITE}

Il n'existe pas une seule définition du système de management sécurité et les définitions existantes sont assez disparates. Nous prenons comme exemple celle qui le définit comme : « ... un dispositif de gestion ne combinant 
personnes, politique, moyens et visant à améliorer les performances d'une entreprise en matière de santé sécurité au travail. C'est un outil qui permet de mieux maîtriser l'organisation de l'entreprise, de progresser en continue en intégrant la SST à toutes les fonctions. ». Ainsi, nous pouvons retenir qu'un SMS englobe l'ensemble d'étapes, d'actions, de processus et de moyens techniques et organisationnels indispensables aux bons fonctionnements et à la gestion de la SST.

\subsection{Les différents risques professionnels}

Il existe plusieurs types ou familles de risques professionnels qui diffèrent les uns des autres par leur nature, leur origine, leurs caractéristiques et leurs conséquences ainsi que par les mesures de prévention qu'ils nécessitent. Par exemple, le risque électrique n'a rien à avoir avec les risques chimiques ou avec le risque de surdité et les mesures de prévention sont différentes les unes des autres.

\subsubsection{Les risques mécaniques}

Nombreux et variés, ils sont présents partout, dans toutes les activités humaines. Ils ont pour origine les déplacements des corps qui par suite de leurs mouvements, possèdent une énergie susceptible d'agresser les hommes. Si ce risque s'explique par les mouvements des objets, il existe également lorsque ce sont les hommes qui bougent, l'objet restant immobile ou en mouvement. Un marteau qui s'abat sur un doigt peut l'écraser ; des engrenages en mouvement peuvent entraîner la main ou les cheveux ; une lame de scie peut couper la chair. Bien qu'il s'agisse ici d'un phénomène physique, par son importance, par la nature des risques et par les mesures de prévention à mettre en œuvre, les risques mécaniques seront étudiés séparément des autres risques physiques.

\subsubsection{Les risques physiques}

Sous cette appellation sont groupés plusieurs risques ayant pour origine des phénomènes physiques variés, souvent très différents les uns des autres et faisant appel à des mesures de prévention distinctes.

- Les risques dus aux vibrations mécaniques ; beaucoup de machines, outils portatifs et véhicules vibrent. Ces vibrations transmises au corps humain portent atteinte à la santé.

- Les risques de surdité dus aux bruits à des niveaux élevés produits par les installations industrielles notamment.

- Les risques électriques qui se traduisent par les électrocutions et les électrisations accidentelles. 
- Les risques dus aux rayonnements ionisants comme la radioactivité ou bien les rayons $\mathrm{X}$ qui conduisent à des atteintes graves de la santé.

- Les risques dus aux rayonnements non ionisants comme les infrarouges ou les rayons lasers qui peuvent conduire à des brûlures.

- Les autres risques physiques dus à des pressions atmosphériques élevées ou faibles, ou encore aux travaux dans les mines.

\subsubsection{Les risques de manutentions manuelle et mécanique}

Les manutentions sont à l'origine de nombreux risques qui se traduisent par des accidents et des maladies professionnelles. Les manutentions manuelles sont encore courantes, malgré l'automatisation et la robotisation de nombre d'entre elles comme les déplacements, les levages, les transports, etc. Dans les deux cas, les risques existent et conduisent à des troubles musculosquelettiques (TMS) ainsi qu'à des accidents mécaniques.

\subsubsection{Les risques chimiques}

Ils s'expliquent par la présence de produits chimiques plus ou moins dangereux, toxiques ou inflammables, dont l'absorption par le corps humain se traduit par des intoxications accidentelles ou chroniques ainsi que par des incendies et explosions.

Les risques chimiques sont fréquents et on les rencontre pratiquement dans toutes les activités humaines, tant en milieu professionnel que chez soi ou pendant les loisirs.

\subsubsection{Les risques biologiques}

Ils sont dus à des micro-organismes pathogènes vivants qui, introduits dans le corps humain, induisent des maladies dont certaines peuvent être très graves comme les hépatites virales. Ces risques nécessitent des mesures de prévention spécifiques. On rencontre les risques biologiques dans de nombreuses activités, souvent inattendues, comme les blanchisseries industrielles ou encore les laboratoires toxicologiques.

\subsubsection{Les risques de circulation et de transport}

Les salariés se déplacent fréquemment tant dans leurs ateliers que dans leurs entreprises ainsi que sur la voie publique. Ces déplacements sont à l'origine de chutes de plain-pied ou de dénivelés, de glissades, de chocs ou d'autres blessures qui sont des accidents du travail. Les accidents de la route sont également considérés sous certaines conditions comme ayant un caractère professionnel. 


\subsubsection{Les autres risques}

Sous cette rubrique, on peut grouper un certain nombre de situations dangereuses particulières à différentes activités, comme celles rencontrées sur les chantiers de bâtiments et de travaux publics ou encore des troubles psychosociaux comme le stress, qui ont une influence certaine sur l'accidentabilité des salariés ${ }^{3}$.

\subsection{Les conséquences}

La protection insuffisante contre l'ensemble des risques vu précédemment et la cause de nombreux accidents de travail, de maladies professionnelles qui donnant suite à des incapacités du travail.

\subsubsection{Les accidents de travail}

Le Dahir $n^{\circ}$ 1-60-223 du 12 ramadan 1382 (6 février 1963) portant modification en la forme du dahir du 25 hija 1345 (25 juin 1927) relatif à la réparation des accidents du travail, définit les accidents du travail comme ceux dont sont victimes les personnes appartenant aux catégories définies aux articles 7 à 12 du même Dahir, et qui donnent droit au profit de la victime ou de ses représentants à une indemnité à la charge de l'entreprise ou de l'employeur, si ces accidents sont survenus par le fait ou à l'occasion du travail ${ }^{4}$.

En fonction de la gravité des lésions, on distingue quatre types d'accidents du travail, correspondant chacun à des modes de réparation spécifiques.

Les accidents du travail sans arrêt qui sont généralement bénins et qui peuvent être soignés sur place, à l'infirmerie de l'entreprise de préférence et qui ne nécessitent que quelques heures de repos ou de soins. Ces accidents ne sont pas à déclarer obligatoirement, mais doivent être consignés sur des registres spéciaux.

Il s'agit de petites blessures (coupures, égratignures, chocs et traumatismes bénins), de très légères intoxications et de petites projections de produits agressifs sur la peau occasionnant des brûlures très superficielles.

Les accidents du travail avec arrêt, de quelques jours à plusieurs mois. Il s'agit d'incapacités temporaires (IT), indemnisées en fonction de la durée de l'arrêt du travail et jusqu'à reprise totale ou partielle du travail. Ce sont des accidents plus graves, nécessitant des soins médicaux ou hospitaliers prolongés et intensifs ainsi qu'un repos de plusieurs jours au moins. Une fracture des os, une entorse, des brûlures importantes mais qui peuvent être soignées sans laisser de séquelles permanentes sont considérées comme des incapacités temporaires et réparées comme telles. 
Les accidents du travail avec incapacité permanente (IP), correspondant à des lésions définitives et des séquelles susceptibles de réduire la capacité de travail.

En fonction de la gravité des dommages corporels, il existe plusieurs taux d'incapacité permanente, se traduisant par des indemnisations (rentes) suivant un barème défini par des textes réglementaires. Un doigt coupé, un œil crevé, une jambe déformée, un poumon partiellement abîmé font l'objet d'indemnisations dont les montants sont variables.

Les accidents du travail mortels avec décès immédiat ou différé, suite à des complications issues d'accidents. Dans ce cas ce sont les ayants droit qui reçoivent les rentes viagères, suivant des règles précises définies par des textes réglementaires.

\subsubsection{Maladies professionnelles}

L'article 2 du dahir du 31 mai 1943 définit les maladies professionnelles comme étant «les manifestations morbides, infectieuses, microbiennes et affections dont la liste est fixée par arrêté du ministre du Travail et des questions sociales pris après avis du ministère de la Santé». Ces maladies peuvent toucher un ou plusieurs organes comme les poumons, le foie, les reins, le système nerveux, la vision, l'ouïe, le sang, les systèmes musculosquelettiques, etc.

La nature de ces maladies est aussi diverse : asthme, anémie, dermatose, encéphalopathie, surdité, atteinte rénale, cancers, etc.

Il existe actuellement 95 tableaux de maladies professionnelles relatives à plusieurs pathologies numérotées de 1 à 86 (avec des bis et des ter) et se répartissent comme suit :

- Agent chimique : 68 tableaux

- Agent biologique : 17 tableaux

- Agent physique : 7 tableaux

- Agent divers : 3 tableaux

Une même nuisance correspondant à un tableau peut conduire dans certains cas à plusieurs maladies (l'amiante provoque plusieurs atteintes à la santé : asbestose, lésions pleurales, mésothéliomes, cancers bronchopulmonaires).

Chaque tableau numéroté concerne les affections correspondant à une nuisance, phénomène ou produit, ou à une famille de nuisances comme les vibrations ou les solvants.

\section{IMPORTANCE DE LA SECURITE DANS LE TRAVAIL}

Dans tous les métiers, les travailleurs peuvent être exposés à une multitude de risques sur le lieu de travail. Les mesures d'hygiène et de sécurité s'attaquent à tout l'éventail des dangers présents sur le lieu de travail, de la 
prévention des accidents à des dangers plus insidieux. Les programmes de santé et de sécurité doivent viser à prévenir les maladies et accidents liés au travail plutôt qu'à résoudre les problèmes a posteriori ${ }^{5}$.

\subsection{Pour l'entreprise}

Il est très important de prendre en compte la prévention des risques au sein des entreprises, et surtout pas oublier ses enjeux économiques, humains et juridiques.

Dans le côté économique, il faut noter que les accidents du travail et maladies professionnelles entrainent des coûts supportés par l'entreprise. La démarche de la prévention diminue les coûts directs supportés au travers des cotisations de sécurité sociale.

En plus des cotisations à la sécurité sociale, d'autre coût s'ajoute d'une manière indirecte, comme le temps passé à secourir la personne, le temps perdu par l'ensemble des salariés après l'accident, le matériel endommagé, le temps passé pour les formalités, autant d'exemple qui viennent perturber la productivité des salariés.

La démarche de prévention des risques garantit la santé des travailleurs et améliore leurs conditions de travail. Les enjeux humains d'une telle démarche sont de plusieurs ordres. La santé des travailleurs est également un élément de performance. Travailler dans de bonnes conditions et en bon état de santé permet aux salariés d'augmenter leur efficacité au service de l'entreprise.

Enfin la démarche de prévention des risques s'inscrit dans le respect de la législation en matière de santé et sécurité au travail. En cas de non-respect de cette législation, l'employeur risque plusieurs sanctions tant en matière financière que pénale.

\subsubsection{Evaluation des risques}

Au vu des enjeux discutés à la première section de cet article, il est de l'intérêt de l'entreprise de s'inscrire dans une démarche de prévention. Elle est tenue par des obligations de l'inspection de travail dans le but de respecter le code du travail.

Afin de veiller à la santé et à la sécurité des salariés l'entreprise doit missionnés plusieurs acteurs et pas seulement une seule personne, et pour aussi mettre en avant la prise de conscience des enjeux de la sécurité.

Le chef d'entreprise est obligé d'assurer la sécurité et de protéger la santé physique et mentale des travailleurs de l'établissement (selon les articles 281 à $301 \mathrm{du}$ code du travail marocain), il doit aussi mettre en œuvre des principes généraux de prévention des risques professionnels et aussi les actions d'information et de formation. On note aussi l'obligation d'avoir un service médical au sein de l'entreprise (selon l'Article 304 et 305 du code du 
travail marocain) pour l'ensemble de ses salariés afin de faire des visites médicales d'embouche, de reprise, de surveillance médicale particulière.

D'autre part l'entreprise qui dispose d'au moins cinquante salariés et obliger de créer un comité de sécurité et d'hygiène (selon le chapitre cinq du code du travail marocain) qui est chargé notamment de :

- Détecter les risques professionnels auxquels sont exposés les salariés de l'entreprise ;

- Assurer l'application des textes législatifs et réglementaires concernant la sécurité et l'hygiène ;

- Veiller au bon entretien et au bon usage des dispositifs de protection des salariés contre les risques professionnels ;

- Veiller à la protection de l'environnement à l'intérieur et aux alentours de l'entreprise ;

- Susciter toutes initiatives portant notamment sur les méthodes et procédés de travail, le choix du matériel, de l'appareillage et de l'outillage nécessaires et adaptés au travail ;

- Présenter des propositions concernant la réadaptation des salariés handicapés dans l'entreprise ;

- Donner son avis sur le fonctionnement du service médical du travail ;

- Développer le sens de prévention des risques professionnels et de sécurité dans l'entreprise ;

- La connaissance de tous les risques afférents au chantier permet de supprimer les dangers et donc de réduire les accidents du travail et les maladies professionnelles.

\subsection{Côté des salariés}

C'est à l'article L. 4122-1 du Code du travail qu'est définie l'obligation générale des salariés en matière de santé et de sécurité au travail: "Conformément aux instructions qui lui sont données par l'employeur, dans les conditions prévues au règlement intérieur pour les entreprises tenues d'en élaborer un, il incombe à chaque travailleur de prendre soin, en fonction de sa formation et selon ses possibilités, de sa santé et de sa sécurité ainsi que de celles des autres personnes concernées par ses actes ou ses omissions au travail".

\subsubsection{Sécurité individuelle et collective}

La sécurité sur le lieu de travail n'est pas seulement une affaire individuelle. L'espace de travail individuel fait partie d'un espace collectif organisé, de ce fait les salariés se doivent d'être vigilants pour eux même mais aussi pour les autres. Nettoyer et ranger leur lieu de travail fait partie de leur 
mission de sécurité au quotidien. Les risques de chutes, surtout en hauteur, pourront être évités pour eux même et les personnes qui gravitent autour d'eux.

Ils doivent signaler toute situation qui selon eux représente un risque grave et imminent. Quand cette décision est correctement justifiée, ils peuvent exercer leur droit de retrait et prévenir un responsable, car selon l'article 287 du code du travail marocaine il est interdit à l'employeur de permettre à ses salariés l'utilisation de produits ou substances, d'appareils ou de machines qui sont reconnus par l'autorité compétente comme étant susceptibles de porter atteinte à leur santé ou de compromettre leur sécurité.

De même, il est interdit à l'employeur de permettre à ses salariés l'utilisation, dans des conditions contraires à celles fixées par voie réglementaire, de produits ou substances, d'appareils ou de machines susceptibles de porter atteinte à leur santé ou de compromettre leur sécurité.

Quant à la sécurité individuelle, elle se traduit par le port des équipements de protection individuels (EPI). Chaussures de sécurité, casque, gants, lunettes de protection ...., chaque salariés est dans l'obligation de les porter en fonction du poste qu'il occupe. En cas de non port de ces EPI, l'incapacité temporaire pourrait devenir une incapacité permanente.

\subsubsection{Rôle et responsabilité au sein d'une organisation}

Les salariés deviennent des acteurs de leur propre sécurité et participent à l'amélioration des conditions de travail auxquelles ils sont confrontés chaque jour, avec leur implication dans la politique de sécurité de l'entreprise qui se considère comme des responsabilités supplémentaires pour eux même.

Chacun du personnel doit bien comprendre qu'il a un rôle à jouer dans la mission de sécurité, et que l'intérêt individuel participe à l'intérêt collectif.

\subsection{L'accompagnement des entreprises}

Dans le cadre de leur mission de conseil (et non de contrôle), les médecins du travail peuvent aider à améliorer l'hygiène, la sécurité et les conditions de travail d'une entreprise afin de prévenir les pathologies en relation avec l'emploi.

Ils accompagnent l'entreprise dans l'identification de ces risques sur les différents postes de travail de l'établissement, afin de mieux adapter la surveillance médicale de votre personnel.

Ils sont formés spécifiquement à la connaissance des risques professionnels.

\subsubsection{Médecin de travail}

La médecine du travail désigne à la fois la spécialité médicale qui concerne la prévention des atteintes à la santé des travailleurs (accidents du 
travail, maladies professionnelles), et la mise en œuvre concrète de cette spécialité dans des services de médecine du travail, appelés services de santé au travail. Ce service a pour mission la préservation de la santé physique et mentale des salariés qu'il prend en charge et accompagne l'entreprise dans le sens de sa politique de sécurité.

Le médecin du travail a un rôle préventif qui consiste à procéder sur les salariés aux examens médicaux nécessaires, notamment à l'examen médical d'aptitude lors de l'embauchage et à éviter toute altération de la santé des salariés du fait de leur travail, notamment en surveillant les conditions d'hygiène dans les lieux de travail, les risques de contamination et l'état de santé des salariés. Il peut aussi donner exceptionnellement, ses soins en cas d'urgence, à l'occasion d'accidents ou de maladies survenus dans l'établissement ainsi qu'à tout salarié victime d'un accident du travail lorsque l'accident n'entraîne pas une interruption du travail du salarié (selon l'article 318 et 319 du code du travail marocain).

\subsubsection{La caisse nationale de sécurité social (CNSS)}

La CNSS à un rôle très important à tenir et son implication est forte dans le développement du pays. Entreprise citoyenne, elle porte haut les valeurs d'égalité pour tous dans le respect de la protection sociale.

Elle assume pleinement ses responsabilités en communicant régulièrement auprès du salarié, à travers différentes campagnes d'information et sur le terrain.

Elle accompagne les entreprises dans une démarche de sécurité de ses salariés dans l'approche sociale, de manière à rembourser les salariés en cas d'accident de travail ou maladie professionnelles.

\section{LA METHODOLOGIE DE TRAVAIL}

\section{Mise en place du système de sécurité}

Afin d'améliorer continûment la Santé et la Sécurité au travail, en y impliquant l'ensemble des niveaux (de la direction au personnel), l'entreprise peut mettre en place un système de management. Il permettra à l'employeur de s'appuyer sur un référentiel et d'intégrer ces domaines à son organisation interne.

\section{Politique de santé et de sécurité au travail}

$\mathrm{Vu}$ la certification de la station de conditionnement de cette société agricole par IFS et BRC, l'unité est arrivée à des niveaux plus avancer de côté sécurité de personnel, car l'hygiène de personnel et la gestion des ressources humaine est très exiger par ces deux certifications.

La volonté de la direction d'installer un système de sécurité ne vise pas l'obtention d'une certification comme OHSAS 18001 par exemple, mais de 
continuer ce qui a été déjà fait lors de la certification IFS et BRC et puis sécuriser le personnel.

C'est pour ça la direction a présenté :

$\checkmark$ Son engagement pour l'amélioration continue ;

$\checkmark$ Son engagement à satisfaire au minimum à la législation en vigueur en matière de santé et de sécurité au travail ;

$\checkmark$ Qu'elle soit consignée par écrit, mise en œuvre et tenue à jour ;

$\checkmark$ Qu'elle soit communiquée à tout le personnel en tentant d'attirer l'attention des employés sur leurs obligations individuelles concernant la santé et la sécurité au travail ;

$\checkmark$ Qu'elle soit disponible pour les parties intéressées ;

$\checkmark$ Qu'elle soit revue régulièrement pour s'assurer qu'elle reste pertinente et appropriée pour l'organisme.

\section{Planification}

L'entreprise soit découpée en plusieurs ensembles, appelés « unités de travail », et regroupant des salariés exposés à des risques similaires ou à des conditions homogènes d'exposition aux risques professionnels.

Les critères discriminants sont : le poste, le secteur d'activité, le métier ou la fonction, les tâches à effectuer, le secteur géographique. 
Tableau 1 : Unités de travail de service emballage

\begin{tabular}{|c|l|c|}
\hline $\mathbf{N}^{\circ}$ & \multicolumn{1}{|c|}{ Intitulé des unités de travail } & $\begin{array}{c}\text { Effectifs } \\
\text { concernés ou } \\
\% \text { d'activité }\end{array}$ \\
\hline $\mathrm{A}$ & $\begin{array}{l}\text { Stockage } \\
\text { (stockage des caisses d'emballage d'asperge dans un magasin) }\end{array}$ \\
\hline $\mathrm{B}$ & $\begin{array}{l}\text { Montage } \\
\text { (montage des caisses d'emballage) }\end{array}$ & $\mathbf{4}$ \\
\hline
\end{tabular}

Tableau 2 : Unités de travail de service réception

\begin{tabular}{|c|c|c|}
\hline $\mathbf{N}^{\circ}$ & Intitulé des unités de travail & $\begin{array}{c}\text { Effectifs } \\
\text { concernés ou } \\
\% \text { d'activité }\end{array}$ \\
\hline $\mathrm{A}$ & $\begin{array}{l}\text { Déchargement } \\
\text { (déchargement des remorques qui transporte le produit récolté du champ) }\end{array}$ & 2 \\
\hline $\mathrm{B}$ & $\begin{array}{l}\text { Lavage } \\
\text { (enlever le sable de produit récolté) }\end{array}$ & 4 \\
\hline $\mathrm{C}$ & $\begin{array}{l}\text { Pesage } \\
\text { (pesage de la quantité réceptionné) }\end{array}$ & 2 \\
\hline $\mathrm{D}$ & $\begin{array}{l}\text { Trempage } \\
\text { (trempage des asperges dans un bassin d'eau chloré) }\end{array}$ & 2 \\
\hline $\mathrm{E}$ & $\begin{array}{l}\text { Hydrocooling } \\
\text { (l'eau froide entre } 1 \text { et } 2{ }^{\circ} \mathrm{C} \text { avec chlore) }\end{array}$ & 2 \\
\hline $\mathrm{F}$ & $\begin{array}{l}\text { Mise à la chambre froide } \\
\text { (mettre le produit à la chambre froide pour garantir la température de conservation) }\end{array}$ & 2 \\
\hline
\end{tabular}


Tableau 3 : Unités de travail de service la salle blanche

\begin{tabular}{|c|l|c|}
\hline $\mathbf{N}^{\circ}$ & \multicolumn{1}{|c|}{ Tntitulé des unités de travail } & \multicolumn{1}{|c|}{\begin{tabular}{c}
\multicolumn{1}{|c}{ Effectifs concernés ou } \\
$\%$ \\
d'activité
\end{tabular}} \\
\hline $\mathrm{A}$ & $\begin{array}{l}\text { Déstockage } \\
\text { (apporter la quantité de produit à conditionner pour chaque chaine) }\end{array}$ & $\mathbf{1}$ \\
\hline $\mathrm{B}$ & $\begin{array}{l}\text { Trempage par caisse } \\
\text { (deuxième rinçage rapide de pré-conditionnement de chaque caisse d'asperge) }\end{array}$ & $\mathbf{2}$ \\
\hline $\mathrm{C}$ & $\begin{array}{l}\text { Etalage et Coupage } \\
\text { (l'alignement des asperges mise sur la chaine de conditionnement afin de passer dans la machine de coupage pour } \\
\text { ajuster chaque asperge) }\end{array}$ & $\mathbf{6}$ \\
\hline $\mathrm{D}$ & $\begin{array}{l}\text { Classification et triage } \\
\text { (sélection des asperges selon le calibre et la couleur) }\end{array}$ & $\mathbf{2 8}$ \\
\hline $\mathrm{E}$ & $\begin{array}{l}\text { Emballage } \\
\text { (mettre le produit dans des caisses de } 2 \mathrm{Kg}, 5 \mathrm{Kg} \text { ou }(8 \text { botte } \mathrm{x} 0.5 \mathrm{~kg}) \text { ou }(8 \times 1 \mathrm{Kg}) \text { ou dans des barquettes selon la } \\
\text { commande du client) }\end{array}$ & $\mathbf{6 0}$ \\
\hline
\end{tabular}

\section{Tableau 4 : Unités de travail de service palettisation}

\begin{tabular}{|c|l|c|}
\hline $\mathrm{N}^{\circ}$ & \multicolumn{1}{|c|}{ Intitulé des unités de travail } & $\begin{array}{c}\text { Effectifs concernés ou } \\
\% \text { d'activité }\end{array}$ \\
\hline $\mathrm{A}$ & $\begin{array}{l}\text { Hydrocooling par caisse } \\
\text { ('eau froide entre } 1 \text { et } 2{ }^{\circ} \mathrm{C} \text { avec chlore) }\end{array}$ & $\mathbf{2}$ \\
\hline $\mathrm{B}$ & $\begin{array}{l}\text { Couverture des caisses } \\
(\text { mettre des couvertures pour les caisses d'asperge de } 2 \mathrm{Kg} \text { et } 5 \mathrm{Kg})\end{array}$ & $\mathbf{1}$ \\
\hline $\mathrm{C}$ & $\begin{array}{l}\text { Trie par caisse } \\
\text { (organisation des caisses par catégorie) }\end{array}$ & $\mathbf{3}$ \\
\hline $\mathrm{D}$ & $\begin{array}{l}\text { Cerclage } \\
\text { (confection d'une palette de caisse d'asperge) }\end{array}$ & $\mathbf{2}$ \\
\hline
\end{tabular}

Tableau 5 : Unités de travail de service expédition

\begin{tabular}{|c|l|c|}
\hline $\mathbf{N}^{\circ}$ & \multicolumn{1}{|c|}{ Intitulé des unités de travail } & $\begin{array}{c}\text { Effectifs concernés ou } \\
\text { \% d'activité }\end{array}$ \\
\hline $\mathrm{A}$ & $\begin{array}{l}\text { Pesage et création de fiche palette } \\
\text { (construction d'une palette de caisse d'asperge) }\end{array}$ & $\mathbf{1}$ \\
\hline $\mathrm{B}$ & $\begin{array}{l}\text { Mise en poche plastique } \\
\text { (aspiration de l'aire de l'intérieur de la palette et le remplacé par le CO2) }\end{array}$ & $\mathbf{2}$ \\
\hline $\mathrm{C}$ & $\begin{array}{l}\text { Chargement } \\
\text { (chargement de produit fini dans les camions destiné à l'export }\end{array}$ & $\mathbf{1}$ \\
\hline
\end{tabular}




\section{Mise en œuvre et fonctionnement}

L'établissement des dangers significatif pour la maitrise repose sur l'évaluation de la gravité et de la fréquence d'exposition des dangers.

Le raisonnement suivi pour la détermination des dangers significatif est présenté dans le tableau 6 et seule les dangers qui dépassent la note 6 , est considérer comme danger significatif.

\section{Gravité :}

- Très grave : mort

- Grave : Blessure ou maladie long arrêt de travail

- Moyen : Blessure avec un court arrêt de travail

- Faible : Blessure sans arrêt de travail

\section{Fréquence d'exposition :}

- Très rare : Jamais survenu dans l'entreprise

- Rare : Arrivé une fois dans l'historique de l'entreprise

- Fréquent : Plusieurs fois dans l'année

- Très fréquent : Quotidien ou Hebdomadaire

\begin{tabular}{|c|c|c|c|c|c|}
\hline \multicolumn{6}{|c|}{ Gravité du domage } \\
\hline & | & & & & \\
\hline Très grave & 8 & 10 & 12 & 16 & \\
\hline Grave & 4 & 6 & 10 & 12 & \multirow{4}{*}{$\begin{array}{l}\text { Exposition } \\
\text { du salarié } \\
\text { au danger }\end{array}$} \\
\hline Moyen & 2 & 4 & 6 & 8 & \\
\hline Faible & 1 & 2 & 3 & 4 & \\
\hline & $\begin{array}{l}\text { très improbable } \\
\text { ou très rare }\end{array}$ & $\begin{array}{l}\text { imporobable } \\
\text { ou rare }\end{array}$ & $\begin{array}{l}\text { probable } \\
\text { ou fréquent }\end{array}$ & $\begin{array}{l}\text { très probable } \\
\text { ou très fréquent }\end{array}$ & \\
\hline
\end{tabular}


Tableau 6: Analyse

des risques

Service : EMBALAGE

\begin{tabular}{|c|c|c|c|c|c|c|c|}
\hline Unité & $\begin{array}{l}\text { Phase } \\
\text { de } \\
\text { travail }\end{array}$ & DANGER & CAUSE & $\begin{array}{l}\text { Mesures de } \\
\text { Prévention }\end{array}$ & Gravité & Fréquence & $\begin{array}{c}\text { Niveau } \\
\text { de } \\
\text { risque }\end{array}$ \\
\hline \multirow{4}{*}{ 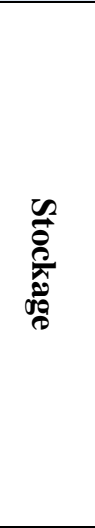 } & \multirow[t]{3}{*}{$\begin{array}{l}\text { Déchargement } \\
\text { des camions / plate- } \\
\text { forme } \\
\text { de caisse avec } \\
\text { chariot élévateur }\end{array}$} & $\begin{array}{l}\text { Chute de la } \\
\text { palette }\end{array}$ & $\begin{array}{l}\text { - Mauvaise positionnement du chariot } \\
\text { élévateur } \\
\text { - Démarrage du camion au moment du } \\
\text { déchargement } \\
\text { - Hauteur de charge de la palette } \\
\text { - Mauvaise montage de la palette }\end{array}$ & Sensibilisation & Faible & Fréquent & 3 \\
\hline & & \multirow{2}{*}{$\begin{array}{l}\text { Renversement } \\
\text { du chariot } \\
\text { élévateur } \\
\end{array}$} & Mauvaise conduite & Formation cariste & Faible & Rare & 2 \\
\hline & & & Mauvais état du matériel & Maintenance matériel & Faible & Rare & 2 \\
\hline & $\begin{array}{l}\text { Stockage des caisses } \\
\text { dans le magasin }\end{array}$ & $\begin{array}{l}\text { Chute des } \\
\text { caisses }\end{array}$ & $\begin{array}{l}\text { - Disposition inadéquate des colis sur } \\
\text { la palette } \\
\text { - Gerbage mal positionné } \\
\text { - Palette cassée / mauvais état }\end{array}$ & Formation cariste & Faible & Fréquent & 3 \\
\hline \multirow{2}{*}{ 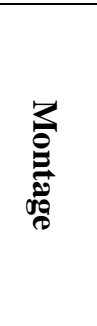 } & $\begin{array}{l}\text { Montage automatique } \\
\text { des colis }\end{array}$ & $\begin{array}{l}\text { Chute des } \\
\text { cartons }\end{array}$ & $\begin{array}{l}\text { - Mauvais disposition des cartons sur } \\
\text { la palette } \\
\text { - Les voies de circulation étroite } \\
\text { - Les voies de circulation encombrées } \\
\text { - Les cartons mal conditionné }\end{array}$ & $\begin{array}{l}\text { Largeur de la voie }>2 \\
\mathrm{~m}\end{array}$ & Faible & Fréquent & 3 \\
\hline & $\begin{array}{c}\text { Montage manuel des } \\
\text { cartons }\end{array}$ & Blessure mains & Absence de gants & $\begin{array}{l}\text { (EPI : équipements } \\
\text { de protection } \\
\text { individuel) }\end{array}$ & Moyen & Rare & 4 \\
\hline
\end{tabular}




\section{Service : RECEPTION}

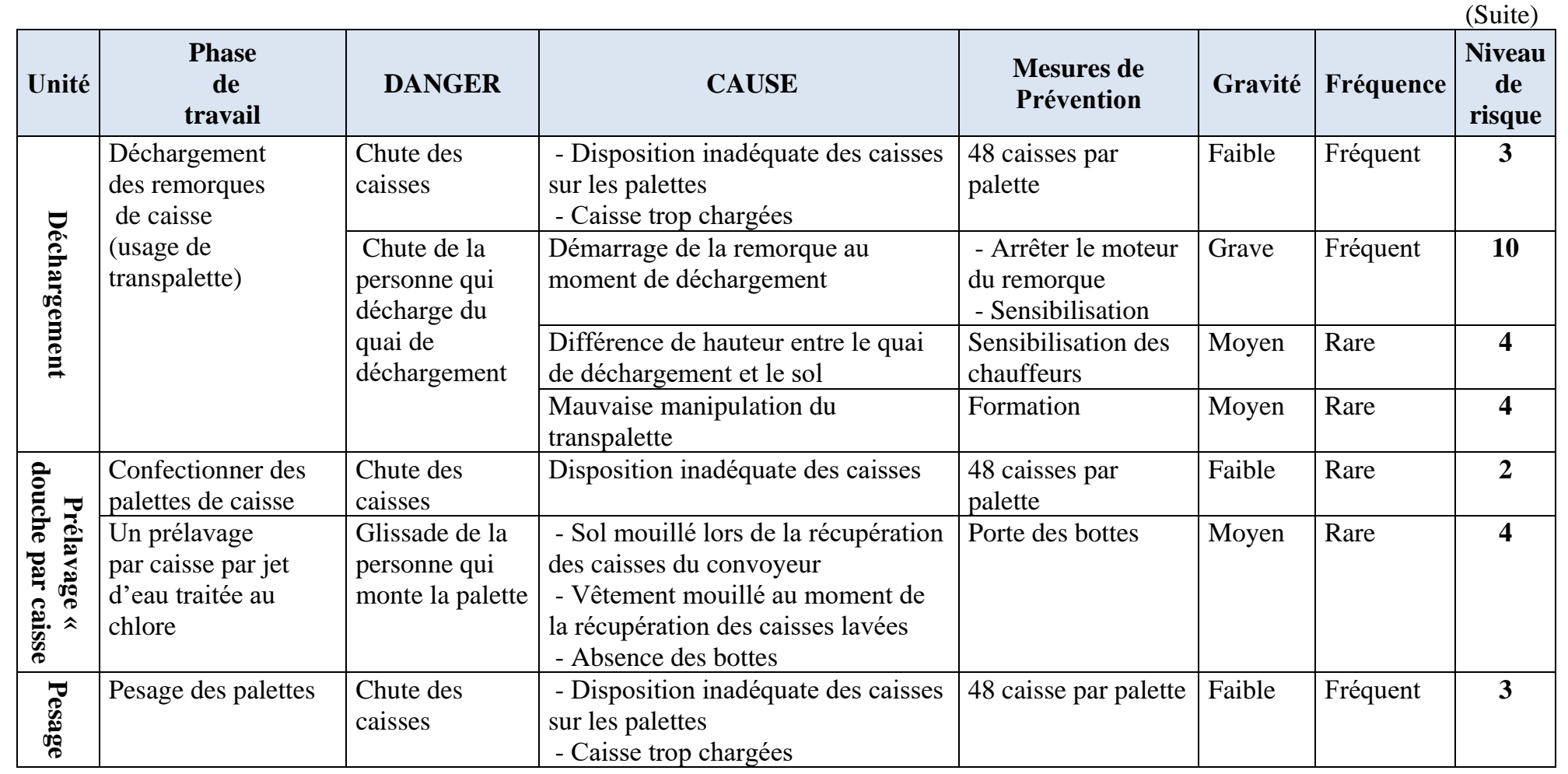




\section{Service : RECEPTION}

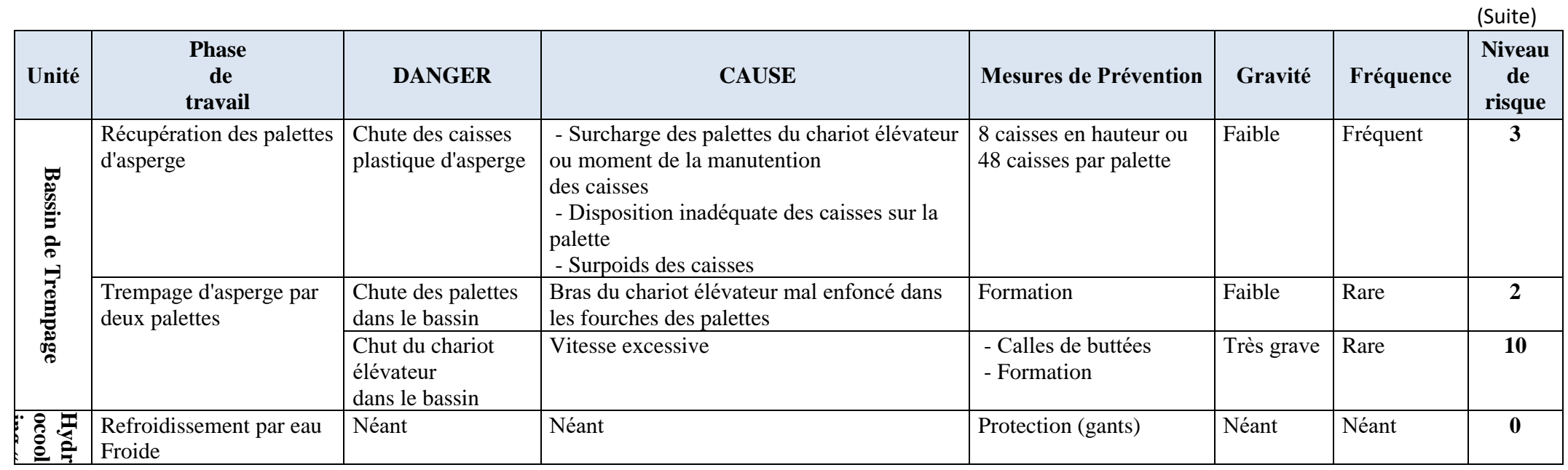

\section{Service : RECEPTION}

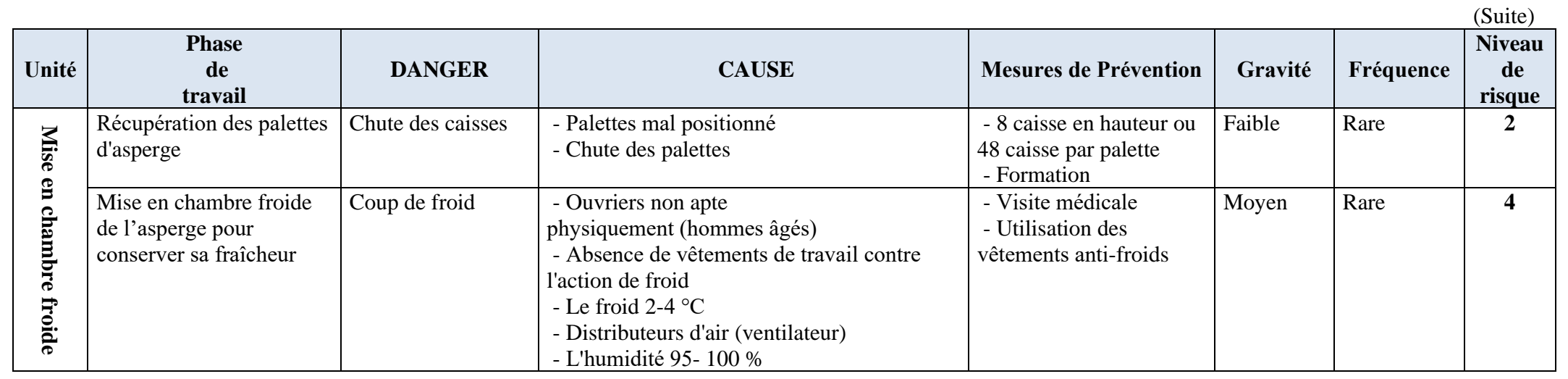




\section{Service : LA SALLE BLANCE (SALLE DE}

\section{CONDITIONNEMENT}

\begin{tabular}{|c|c|c|c|c|c|c|c|}
\hline Unité & $\begin{array}{l}\text { Phase } \\
\text { de } \\
\text { travail }\end{array}$ & $\begin{array}{l}\text { DANGE } \\
\quad \text { R }\end{array}$ & CAUSE & Mesures de Prévention & Gravité & $\begin{array}{c}\text { Fréquenc } \\
\text { e }\end{array}$ & $\begin{array}{c}\text { (Suite) } \\
\text { Nivea } \\
\mathbf{u} \\
\text { de } \\
\text { risque }\end{array}$ \\
\hline \multirow{2}{*}{ 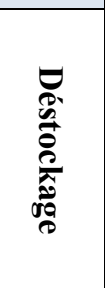 } & $\begin{array}{l}\text { Sortir des palettes de la } \\
\text { chambre froide }\end{array}$ & $\begin{array}{l}\text { Attraper } \\
\text { froid }\end{array}$ & $\begin{array}{l}\text { - Ouvriers non apte } \\
\text { physiquement (hommes âgés) } \\
\text { - Absence de vêtements de travail } \\
\text { contre l'action de froid }\end{array}$ & $\begin{array}{l}\text { - Visite médicale } \\
\text { - utilisation des } \\
\text { vêtements anti-froids }\end{array}$ & Moyen & Rare & 4 \\
\hline & $\begin{array}{l}\text { Alimenter la salle blanche } \\
\text { (usage de transpalette } \\
\text { manuel) }\end{array}$ & $\begin{array}{l}\text { Chute des } \\
\text { caisses }\end{array}$ & $\begin{array}{l}\text { - Disposition inadéquate des } \\
\text { palettes } \\
\text { - Sol mouillé }\end{array}$ & Eliminer l'excès d'eau & Moyen & Rare & 4 \\
\hline 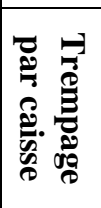 & $\begin{array}{l}\text { Mettre chaque caisse dans } \\
\text { l'eau chlorée 2-5 PPM }\end{array}$ & $\begin{array}{l}- \\
\text { Vêtements } \\
\text { mouillés } \\
\text { - Sole } \\
\text { mouillé }\end{array}$ & $\begin{array}{l}\text { - Absence de vêtements de travail } \\
\text { imperméable } \\
\text { - Débordement du bac }\end{array}$ & $\begin{array}{l}\text { - Vêtement } \\
\text { imperméable (tabliers) } \\
\text { - Cache manche } \\
\text { - Raclette passée } \\
\text { régulièrement }\end{array}$ & Faible & Fréquent & 3 \\
\hline 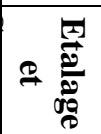 & $\begin{array}{l}\text { Aligner les asperges sur la } \\
\text { chaine pour les faire passer } \\
\text { dans la machine de coupage }\end{array}$ & $\begin{array}{l}\text { Choc } \\
\text { électrique }\end{array}$ & $\begin{array}{l}\text { Poste électrique près de la chaine } \\
\text { de conditionnement }\end{array}$ & $\begin{array}{l}\text { - Panneaux de } \\
\text { signalisation } \\
\text { - Poste fermé à clé }\end{array}$ & $\begin{array}{l}\text { Très } \\
\text { grave }\end{array}$ & Très rare & 8 \\
\hline
\end{tabular}


Service : LA SALLE BLANCE (SALLE DE CONDITIONNEMENT)

\begin{tabular}{|c|c|c|c|c|c|c|c|}
\hline Unité & $\begin{array}{c}\text { Phase } \\
\text { de } \\
\text { travail }\end{array}$ & DANGER & CAUSE & Mesures de Prévention & Gravité & Fréquence & $\begin{array}{c}\text { Niveau } \\
\text { de } \\
\text { risque }\end{array}$ \\
\hline \multirow{5}{*}{ 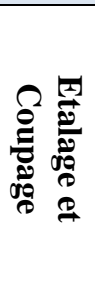 } & \multirow{5}{*}{$\begin{array}{l}\text { Aligner les asperges sur } \\
\text { la chaine pour les faire } \\
\text { passer dans la machine } \\
\text { de coupage }\end{array}$} & \multirow{5}{*}{$\begin{array}{l}\text { Ecrasement des } \\
\text { doigts }\end{array}$} & Manque de vigilance & Sensibilisation & \multirow[t]{5}{*}{ Grave } & \multirow[t]{5}{*}{ Rare } & \multirow[t]{5}{*}{6} \\
\hline & & & Manque de signalisation & Panneaux de signalisation & & & \\
\hline & & & Manque des gants de protection & Distribution des gants & & & \\
\hline & & & $\begin{array}{l}\text { Absence de mécanisme d'arrêt } \\
\text { d'urgence }\end{array}$ & $\begin{array}{l}\text { Mécanisme d'arrêt } \\
\text { d'urgence de la chaine }\end{array}$ & & & \\
\hline & & & $\begin{array}{l}\text { Poste de travail insuffisamment } \\
\text { éclairé pour l'activité exercée }\end{array}$ & Signalisation & & & \\
\hline 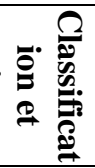 & $\begin{array}{l}\text { Calibrage des asperges } \\
\text { avec convoyeur }\end{array}$ & $\begin{array}{l}\text { - Main coincées } \\
\text { entre convoyeur et } \\
\text { inox } \\
\text { - Blessure mains }\end{array}$ & $\begin{array}{l}\text { - Manque de vigilance } \\
\text { - Vitesse du convoyeur }\end{array}$ & Sensibilisation & Grave & Très rare & 4 \\
\hline \multirow{4}{*}{ 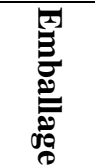 } & \multirow{4}{*}{$\begin{array}{l}\text { Emballage des asperges } \\
\text { selon la commande (en } \\
\text { botte, en vrac ou en } \\
\text { barquettes filmées) }\end{array}$} & \multirow{4}{*}{$\begin{array}{l}\text { - Coupure } \\
\text { - Blessures }\end{array}$} & Manque de vigilance & Sensibilisation & \multirow[t]{4}{*}{ Moyen } & \multirow[t]{4}{*}{ Rare } & \multirow[t]{4}{*}{4} \\
\hline & & & Manque de signalisation & Panneaux de signalisation & & & \\
\hline & & & Utilisation des couteaux & Sensibilisation & & & \\
\hline & & & Utilisation machine thermo soudure & Formation & & & \\
\hline
\end{tabular}




\section{Service : PALLITISATION}

\begin{tabular}{|c|c|c|c|c|c|c|c|}
\hline Unité & $\begin{array}{l}\text { Phase } \\
\text { de } \\
\text { travail }\end{array}$ & DANGER & CAUSE & Mesures de Prévention & Gravité & Fréquence & $\begin{array}{c}\text { Niveau } \\
\text { de } \\
\text { risque }\end{array}$ \\
\hline 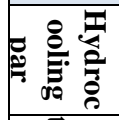 & $\begin{array}{l}\text { Refroidissement par eau } \\
\text { froide (asperges en bottes } \\
\text { et/ou en vrac) }\end{array}$ & Froid aux mains & $\begin{array}{l}\text { - Vêtement mouillé } \\
\text { - Température de l'eau } \\
\text { - Manque EPI }\end{array}$ & $\begin{array}{l}\text { - Vêtements imperméable } \\
\text { - Gants de protection }\end{array}$ & Faible & Rare & 2 \\
\hline 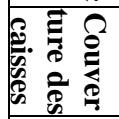 & $\begin{array}{l}\text { Agrafage des couvertures } \\
\text { spéciales pour chaque } \\
\text { catégorie }\end{array}$ & $\begin{array}{l}\text { Blessures avec } \\
\text { agrafes }\end{array}$ & $\begin{array}{l}\text { - Absence des gants de protection } \\
\text { - La rapidité } \\
\text { - Mauvaise utilisation de l'agrafeuse }\end{array}$ & - Gants de protection & Moyen & Rare & 4 \\
\hline \multirow{3}{*}{$\frac{\Omega}{0}$} & $\begin{array}{l}\text { Tri et regroupement des } \\
\text { caisses par catégorie }\end{array}$ & $\begin{array}{l}\text { - Chute des caisses } \\
\text { - chute en hauteur }\end{array}$ & $\begin{array}{l}\text { - Des caisses males rangées) } \\
\text { - Disposition inadéquate des caisses sur la palette }\end{array}$ & Sensibilisation & Faible & Rare & 2 \\
\hline & $\begin{array}{l}\text { Confectionner et finalisation } \\
\text { des palettes d'asperge }\end{array}$ & Chute des caisses & Disposition inadéquate des caisses & Sensibilisation & Faible & Rare & 2 \\
\hline & $\begin{array}{l}\text { Cerclage (machine pour } \\
\text { serrer le feuillard) }\end{array}$ & Blessures & Serrer les palettes en force & Un seul opérateur & Faible & Très rare & 1 \\
\hline
\end{tabular}




\section{Service : EXPEDITION}

\begin{tabular}{|c|c|c|c|c|c|c|c|}
\hline Unité & $\begin{array}{l}\text { Phase } \\
\text { de } \\
\text { travail }\end{array}$ & DANGER & CAUSE & Mesures de Prévention & Gravité & Fréquence & $\begin{array}{l}\text { Niveau } \\
\text { de } \\
\text { risque }\end{array}$ \\
\hline 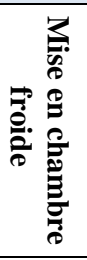 & $\begin{array}{l}\text { Mise en chambre froide de } \\
\text { l'asperge pour } \\
\text { conserver sa fraîcheur }\end{array}$ & Coup de froid & $\begin{array}{l}\text { - Ouvriers non apte } \\
\text { physiquement (hommes âgés) } \\
\text { - Absence de vêtements de travail contre } \\
\text { l'action de froid } \\
\text { - Le froid } 2-4{ }^{\circ} \mathrm{C} \\
\text { - Distributeurs d'air (ventilateur) } \\
\text { - L'humidité } 95 \text { - } 100 \%\end{array}$ & $\begin{array}{l}\text { - Visite médicale } \\
\text { - utilisation des } \\
\text { vêtements anti-froids }\end{array}$ & Moyen & Rare & 4 \\
\hline \multirow{3}{*}{ 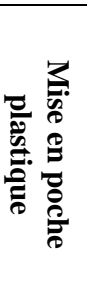 } & $\begin{array}{l}\text { Mettre les palettes en } \\
\text { poches plastique }\end{array}$ & Coup de froid & $\begin{array}{l}\text { - Ouvriers non apte } \\
\text { physiquement (hommes âgés) } \\
\text { - Absence de vêtements de travail contre } \\
\text { l'action de froid }\end{array}$ & $\begin{array}{l}\text { - Visite médicale } \\
\text { - utilisation des } \\
\text { vêtements anti-froids }\end{array}$ & Moyen & Rare & 4 \\
\hline & \multirow{2}{*}{$\begin{array}{c}\text { Traitement au } \mathrm{CO}_{2} \\
\text { aspirer l'air de l'intérieur } \\
\text { de la palette et le remplacé } \\
\text { par le } \mathrm{CO} 2\end{array}$} & Blessure des mains & Utilisation de couteaux & Gants de protection & Moyen & Rare & 4 \\
\hline & & $\begin{array}{l}\text { - Asphyxie } \\
\text { - Explosion des } \\
\text { bouteilles }\end{array}$ & Utilisation de bouteille de $\mathrm{CO} 2$ & $\begin{array}{l}\text { Vérification de l'état des } \\
\text { bouteilles et système } \\
\text { avant l'utilisation }\end{array}$ & Très grave & Très rare & 8 \\
\hline
\end{tabular}


Service : EXPEDITION

\begin{tabular}{|c|c|c|c|c|c|c|c|}
\hline Unité & $\begin{array}{l}\text { Phase } \\
\text { de } \\
\text { travail }\end{array}$ & DANGER & CAUSE & $\begin{array}{l}\text { Mesures de } \\
\text { Prévention }\end{array}$ & Gravité & Fréquence & $\begin{array}{c}\text { Niveau } \\
\text { de } \\
\text { risque }\end{array}$ \\
\hline \multirow{5}{*}{ 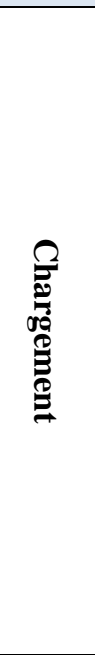 } & $\begin{array}{l}\text { Sortie des palettes } \\
\text { finies de la chambre } \\
\text { froide }\end{array}$ & Coup de froid & $\begin{array}{l}\text { - Ouvriers non apte } \\
\text { physiquement (hommes âgés) } \\
\text { - Absence de vêtements de travail } \\
\text { contre l'action de froid } \\
\text { - Le froid } 2-4^{\circ} \mathrm{C} \\
\text { - distributeurs d'air (ventilateur) } \\
\text { - 1'humidité } 95-100 \% \text {. }\end{array}$ & $\begin{array}{l}\text { - Visite médicale } \\
\text { - utilisation des } \\
\text { vêtements anti-froids }\end{array}$ & Moyen & Rare & 4 \\
\hline & \multirow{4}{*}{$\begin{array}{l}\text { Chargement } \\
\text { des camions } \\
\text { de caisse } \\
\text { (usage de transpalette) }\end{array}$} & Chute des caisses & $\begin{array}{l}\text { - Disposition inadéquate des caisses sur } \\
\text { les palettes } \\
\text { - Caisse trop chargées }\end{array}$ & 48 caisses par palette & Faible & Fréquent & 3 \\
\hline & & \multirow{3}{*}{$\begin{array}{l}\text { Chute de la } \\
\text { personne qui } \\
\text { charge du quai } \\
\text { de chargement }\end{array}$} & $\begin{array}{l}\text { Démarrage de la remorque au moment } \\
\text { de chargement }\end{array}$ & $\begin{array}{l}\text { - Arrêter le moteur } \\
\text { du remorque } \\
\text { - Sensibilisation }\end{array}$ & Grave & Fréquent & 10 \\
\hline & & & $\begin{array}{l}\text { Différence de hauteur entre le quai de } \\
\text { chargement et le sol }\end{array}$ & $\begin{array}{l}\text { Sensibilisation des } \\
\text { chauffeurs }\end{array}$ & Moyen & Rare & 4 \\
\hline & & & Mauvaise manipulation du transpalette & Formation & Moyen & Rare & 4 \\
\hline
\end{tabular}

Notre évaluation des risques actuels a montré des risques significatifs (niveau de risque est supérieur à 6). Pour pallier ces risques décelés à travers ce diagnostic, nous avons proposé un plan d'action qui comporte les actions correctives et précises les responsabilités ainsi que les délais à respecter. 


\section{Tableau 7 : Plan d'action}

\begin{tabular}{|c|c|c|c|c|c|c|c|c|c|c|}
\hline \multirow[b]{2}{*}{ Service } & \multirow[b]{2}{*}{ Unité } & \multirow{2}{*}{$\begin{array}{c}\text { Phase } \\
\text { de } \\
\text { travail }\end{array}$} & \multirow{2}{*}{$\begin{array}{c}\text { Situation } \\
\text { dangereuse } \\
\text { à traiter } \\
\end{array}$} & \multirow{2}{*}{$\begin{array}{c}\text { Origines des } \\
\text { situations } \\
\text { dangereuses }\end{array}$} & \multirow{2}{*}{$\begin{array}{c}\text { Mesure } \\
\text { de } \\
\text { maitrise }\end{array}$} & \multicolumn{2}{|c|}{ Maitrise } & \multirow[b]{2}{*}{ Action } & \multirow[b]{2}{*}{ Pilot } & \multirow[b]{2}{*}{ Délais } \\
\hline & & & & & & Oui & Non & & & \\
\hline \multirow{4}{*}{ 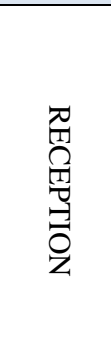 } & \multirow{2}{*}{ 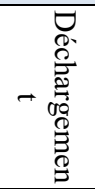 } & \multirow{2}{*}{$\begin{array}{l}\text { Déchargement } \\
\text { des remorques } \\
\text { de caisse } \\
\text { (usage de } \\
\text { transpalette) }\end{array}$} & \multirow{2}{*}{$\begin{array}{l}\text { Chute de la } \\
\text { personne qui } \\
\text { décharge du } \\
\text { quai de } \\
\text { déchargement }\end{array}$} & \multirow{2}{*}{$\begin{array}{l}\text { Démarrage de la } \\
\text { remorque au } \\
\text { moment de } \\
\text { déchargement }\end{array}$} & $\begin{array}{l}\text { Arrêter le } \\
\text { moteur de la } \\
\text { remorque }\end{array}$ & & $\nabla$ & Surveillance & $\begin{array}{l}\text { Responsable des } \\
\text { réceptions }\end{array}$ & Immédiat \\
\hline & & & & & Sensibilisation & & $\nabla$ & Sensibilisation & $\begin{array}{l}\text { Responsable des } \\
\text { réceptions }\end{array}$ & Immédiat \\
\hline & \multirow{2}{*}{ 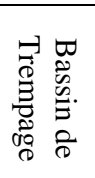 } & \multirow[t]{2}{*}{$\begin{array}{l}\text { Trempage } \\
\text { d'asperge par } \\
\text { deux palettes }\end{array}$} & \multirow{2}{*}{$\begin{array}{l}\text { Chut du } \\
\text { chariot } \\
\text { élévateur } \\
\text { dans le bassin }\end{array}$} & \multirow[t]{2}{*}{ Vitesse excessive } & Calles de buttées & $\nabla$ & & $\begin{array}{l}\text { Vérification au } \\
\text { régulier de } \\
\text { présence } \\
\end{array}$ & $\begin{array}{l}\text { Responsable de } \\
\text { maintenance }\end{array}$ & Immédiat \\
\hline & & & & & Formation & $\nabla$ & & Néant & Néant & Néant \\
\hline \multirow{4}{*}{ 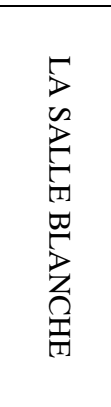 } & \multirow{4}{*}{ 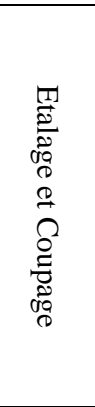 } & \multirow{4}{*}{$\begin{array}{c}\text { Aligner les } \\
\text { asperges sur la } \\
\text { chaine pour les } \\
\text { faire passer dans } \\
\text { la machine de } \\
\text { coupage }\end{array}$} & \multirow[t]{4}{*}{$\begin{array}{l}\text { Écrasement } \\
\text { des doigts }\end{array}$} & $\begin{array}{l}\text { Manque de } \\
\text { vigilance }\end{array}$ & Sensibilisation & $\nabla$ & & Néant & R. Qualité & Néant \\
\hline & & & & $\begin{array}{l}\text { Manque de } \\
\text { signalisation }\end{array}$ & $\begin{array}{l}\text { Panneaux de } \\
\text { signalisation }\end{array}$ & & $\nabla$ & $\begin{array}{l}\text { Identifier les } \\
\text { types de } \\
\text { panneaux à } \\
\text { mettre }\end{array}$ & R. Qualité & 1 mois \\
\hline & & & & $\begin{array}{l}\text { Manque des gants } \\
\text { de protection }\end{array}$ & $\begin{array}{l}\text { Distribution des } \\
\text { gants }\end{array}$ & $\nabla$ & & Néant & Néant & Néant \\
\hline & & & & $\begin{array}{l}\text { Absence de } \\
\text { mécanisme d'arrêt } \\
\text { d'urgence }\end{array}$ & $\begin{array}{l}\text { Mécanisme } \\
\text { d'arrêt d'urgence } \\
\text { de la chaine }\end{array}$ & $\nabla$ & & Néant & Néant & Néant \\
\hline
\end{tabular}




\begin{tabular}{|c|c|c|c|c|c|c|c|c|c|c|}
\hline \multirow[b]{2}{*}{ Service } & \multirow[b]{2}{*}{ Unité } & \multirow{2}{*}{$\begin{array}{c}\text { Phase } \\
\text { de } \\
\text { travail }\end{array}$} & \multirow{2}{*}{$\begin{array}{c}\text { Situation } \\
\text { dangereuse } \\
\text { à traiter }\end{array}$} & \multirow{2}{*}{$\begin{array}{c}\text { Origines des } \\
\text { situations } \\
\text { dangereuses } \\
\end{array}$} & \multirow{2}{*}{$\begin{array}{c}\text { Mesure } \\
\text { de } \\
\text { maitrise }\end{array}$} & \multicolumn{2}{|c|}{ Maitrise } & \multirow[b]{2}{*}{ Action } & \multirow[b]{2}{*}{ Pilot } & \multirow[b]{2}{*}{ Délais } \\
\hline & & & & & & Oui & Non & & & \\
\hline \multirow[b]{2}{*}{ 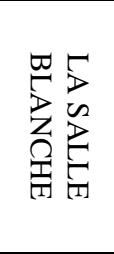 } & \multirow{2}{*}{ 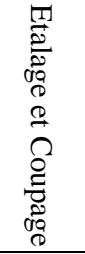 } & \multirow{2}{*}{$\begin{array}{l}\text { Aligner les } \\
\text { asperges sur la } \\
\text { chaine pour } \\
\text { les faire passer } \\
\text { dans la } \\
\text { machine de } \\
\text { coupage }\end{array}$} & \multirow[t]{2}{*}{\begin{tabular}{|l} 
Choc \\
électrique
\end{tabular}} & \multirow{2}{*}{$\begin{array}{l}\text { Poste électrique } \\
\text { près la chaine de } \\
\text { conditionnement }\end{array}$} & $\begin{array}{l}\text { Panneaux de } \\
\text { signalisation }\end{array}$ & $\square$ & & Néant & Néant & Néant \\
\hline & & & & & $\begin{array}{l}\text { Poste fermé à } \\
\text { clé }\end{array}$ & $\nabla$ & & $\begin{array}{l}\text { Vérification au } \\
\text { régulier de la } \\
\text { fermeture }\end{array}$ & $\begin{array}{l}\text { Responsable de } \\
\text { maintenance }\end{array}$ & Immédiat \\
\hline \multirow{3}{*}{ 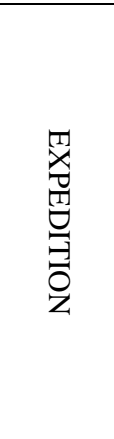 } & 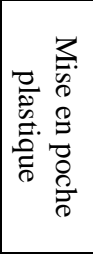 & $\begin{array}{l}\text { Traitement au } \\
\mathrm{CO} 2 \text { aspirer } \\
\text { l'air de } \\
\text { l'intérieur de } \\
\text { la palette et le } \\
\text { remplacé par } \\
\text { le CO2 }\end{array}$ & \begin{tabular}{|l} 
- Asphyxie \\
- Explosion \\
des bouteilles
\end{tabular} & $\begin{array}{l}\text { Utilisation de } \\
\text { bouteille de } \mathrm{CO} 2\end{array}$ & $\begin{array}{l}\text { Vérification de } \\
\text { l'état des } \\
\text { bouteilles et } \\
\text { système avant } \\
\text { l'utilisation }\end{array}$ & $\square$ & & Néant & Néant & Néant \\
\hline & \multirow{2}{*}{ 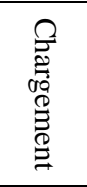 } & \multirow{2}{*}{$\begin{array}{l}\text { Chargement } \\
\text { des camions } \\
\text { de caisse } \\
\text { (usage de } \\
\text { transpalette) }\end{array}$} & \multirow{2}{*}{$\begin{array}{l}\text { Chute de la } \\
\text { personne qui } \\
\text { charge du } \\
\text { quai de } \\
\text { chargement }\end{array}$} & \multirow{2}{*}{$\begin{array}{l}\text { Démarrage de la } \\
\text { remorque au } \\
\text { moment de } \\
\text { chargement }\end{array}$} & \begin{tabular}{|l} 
Arrêter le \\
moteur de la \\
remorque
\end{tabular} & & $\nabla$ & Surveillance & $\begin{array}{l}\text { Responsable des } \\
\text { réceptions }\end{array}$ & Immédiat \\
\hline & & & & & Sensibilisation & & $\nabla$ & Sensibilisation & $\begin{array}{l}\text { Responsable des } \\
\text { réceptions }\end{array}$ & Immédiat \\
\hline
\end{tabular}




\section{RESULTATS ET DISCUSSION}

L'évaluation des risques réalisée nous a permis l'identification de six dangers significatifs :

$>$ Danger 1.: Réception (Chute de la personne qui décharge du quai de déchargement).

$>$ Danger 2.: Réception (Chut du chariot élévateur dans le bassin).

$>$ Danger 3.: La salle blanche (écrasement des doigts).

$>$ Danger 4. : La salle blanche (Choc électrique).

$>$ Danger 5. : Expédition (Asphyxie, explosion des bouteilles de CO2).

$>$ Danger 6. : Expédition (Chute de la personne qui charge du quai de chargement).

Le plan d'action élaboré propose les mesures de maîtrise des dangers identifiés, incluant les procédures de surveillance, les mesures correctives et les procédures de vérification.

Pour une mise en œuvre efficace du système de management de sécurité, nous recommandons les actions suivantes :

$>$ Accomplir les actions correctives proposées dans le plan d'action pour relever les non-conformités décelées suite à l'évaluation des risques pour appuyer le système de management de sécurité

$>$ Valider et mettre en application les mesures de maitrise des dangers significatifs identifiés et élaborer la documentation manquante.

$>$ Etablir, réaliser et documenter les programmes de la formation sur la démarche de l'OHSAS 18001 pour l'ensemble du personnel impliqué.

$>$ Programmer et réaliser la révision et la vérification du système de sécurité afin de valider son bon fonctionnement.

Nous sommes convaincus que l'esprit d'équipe dirigeante et technique de cette société agricole, sa patience et son ambition permettront de relever ce défi. Nous croyons parfaitement en la capacité de l'entreprise à consolider son image de société type dans la région de Gharb, avec son respect de la législation de travail en matière de l'hygiène et de sécurité.

\section{CONCLUSION}

Les démarches de management de la santé et la sécurité au travail visent à améliorer la performance sociale et économique des entreprises grâce à une politique affirmée de maîtrise des risques professionnels et une culture de prévention. Il peut s'agir d'une norme, d'un code, d'un règlement, d'un guide ou de tout texte que l'entreprise se sera fixé comme exigence à satisfaire. Les principaux référentiels les plus utilisés en management de la santé et sécurité au travail sont :

- OHSAS 18001 conçu à l'origine pour la certification par un ensemble d'organismes internationaux privés et publics puis repris sous la forme d'une norme par le British Standard Institute (BSI) 

(OIT).

- ILO-OSH 2001 conçu par l'Organisation Internationale du Travail

La présente étude a abouti à la mise en place du système de management de sécurité, au niveau de la société agricole choisi pour cette étude. L'étude a concerné toutes les étapes de la chaîne de conditionnement d'asperges et a permis d'identifier les dangers significatifs à maitriser en vue de d'assurer la sécurité de personnel.

La réussite de la mise en application passe par la garantie de la conformité du système aux exigences principale du référentiel OHSAS 18001. Le plan d'action élaboré propose les actions correctives à entreprendre à cet égard.

La réussite de la mise en œuvre du système de management de sécurité nécessite la mobilisation de l'équipe pour la mise en application des actions correctives et des mesures de maîtrises proposées et ce dans les meilleurs délais.

\section{References:}

1. AFNOR (2003) : Hygiène des aliments, éléments de maîtrise, méthode HACCP. Page. 432.

2. M.J. Fraqueza ; A.S. Barreto , (2014). HACCP: Hazard Analysis and Critical Control Points. Book Editor(s): Fidel Toldrá Y. H. Hui Iciar Astiasarán Joseph G. Sebranek Règine Talon First.

3. Risques et accidents industriels majeurs, Nichan Margossian, pages 272. Collection Technique et ingénierie, (2006) et Dunod.

4. Dahir n'1-03-194 du 14 rejeb (11 septembre 2003) portant promulgation de la loi $n^{\circ} 65-99$ relative au Code du Travail.

5. FITERMAN Charles - Avis et Rapports du C.E.S - 11/03/2003 - ${ }^{\circ}$ 07/2003 -page 147. 